\title{
Reconfigurable Tangible Devices for 3D Virtual Object Manipulation by Single or Multiple Users
}

\author{
Laurent Aguerreche* \\ Université Européenne de Bretagne \\ INSA, INRIA, IRISA
}

\author{
Thierry Duval ${ }^{\dagger}$ \\ Université Européenne de Bretagne \\ Université de Rennes 1, INRIA, IRISA
}

\author{
Anatole Lécuyer ${ }^{\ddagger}$ \\ INRIA Rennes - Bretagne Atlantique
}

\begin{abstract}
In this paper we introduce the concept of a Reconfigurable Tangible Device for manipulation of 3D objects in virtual environments by single or multiple users. This Reconfigurable Tangible Device (RTD) provides points of manipulation rigidly linked together. The shape of the RTD can be reconfigured at any time as its arms can be compressed or stretched by users at will. Due to its simple shape the Reconfigurable Tangible Device can be attached to any 3D virtual object. Then, it can fully define the motion of the virtual object in 6 Degrees of Freedom. Two examples of Reconfigurable Tangible Device were developed: one with three points of manipulation (a reconfigurable triangle) and one with four points. We illustrate how these two simple devices can match many different shapes of $3 \mathrm{D}$ objects, and in different contexts. Preliminary testing was conducted with the RTD based on three points of manipulation involving a collaborative manipulation task in virtual reality. It produced better subjective appreciation for the RTD compared to more classical 3D collaborative techniques.
\end{abstract}

CR Categories: H.5.2 [Information Interfaces and Presentation]: User Interfaces-Input devices and strategies

Keywords: Virtual Reality, Collaborative Interaction, 3D Interaction, Reconfigurable Tangible User Interface.

\section{Introduction}

$3 \mathrm{D}$ object manipulation is one of the most fundamental tasks to achieve in virtual reality. Many efficient interaction techniques have been developed in this area in the past decade [Bowman et al. 2004]. Few manipulation techniques developed in virtual reality make use of Tangible User Interfaces (TUIs), such as in [Hinckley et al. 1997; Salzmann et al. 2009]. Tangible User Interfaces were designed to give a physical form to digital information [Ullmer and Ishii 2000]. They were shown to improve the manipulation of objects in many cases, in 3D or in 2D applications [Ishii and Ullmer 1997].

In virtual reality, a famous example is the neurosurgery visualization application of Hinckley et al. [Hinckley et al. 1997] in which a user holds two physical objects: a head doll in one hand and a plastic plane in the other hand. These tools are used to select and visu-

\footnotetext{
*e-mail: laurent.aguerreche@ irisa.fr

†e-mail: thierry.duval@irisa.fr

‡e-mail: anatole.lecuyer@irisa.fr
}

alize more directly and more efficiently cuts in a 3D brain model. The Monkey [Esposito et al. 1995] is dedicated to the configuration of virtual human postures for the purpose of computer animation. Note that a TUI does not necessarily have to take the shape of the corresponding real object (iconic representation [Ullmer and Ishii 2000]) but it can also be an abstract representation of this real object (symbolic representation). In [Salzmann et al. 2009], a collaborative tangible user interface is used as a passive link between two users for a $3 \mathrm{D}$ collaborative manipulation in virtual reality.

We can further subdivide tangible user interfaces from the literature into two distinct types: non-reconfigurable TUIs and reconfigurable TUIs. Non-reconfigurable tangible user interfaces have a shape that cannot be modified, whereas reconfigurable TUIs are tangible interfaces whose shape can be modified. A first example of a reconfigurable TUI consists in assembling or removing physical blocks that progressively design and match 3D object shapes. Research on this topic was initiated with architectural applications [Frazer 1995] and led to numerous TUIs such as the MERL bricks [Anderson et al. 1999] or the ActiveCubes [Watanabe et al. 2004]. Instead of building a shape by assembling rigid blocks, another approach consists in deforming a malleable TUI. Users are then able to use pressure to build shapes in 3D modeling tools for instance [Smith et al. 2008]. Several reconfigurable TUIs follow an approach balancing between malleability and rigidity. The Senspectra TUI [LeClerc et al. 2007] connects rigid balls by flexible joints to form an overall flexible structure. The Glume TUI [Parkes et al. 2006] connects malleable balls by a rigid structure. However, all these reconfigurable TUIs were built for specific applications, e.g. games or education, and never in the context of virtual reality and $3 \mathrm{D}$ virtual object manipulation.

Therefore, in this paper, we introduce a novel concept of reconfigurable tangible interface that can match many shapes of 3D objects, for the purpose of object manipulation by single or multiple users in virtual environments. We also provide a preliminary evaluation that is focused on testing rigidity and ease of manipulation for one instance of this concept.

\section{A Reconfigurable Tangible Device for 3D Object Manipulation in Virtual Reality}

\subsection{Concept}

Our main objective is to match numerous shapes of 3D objects, in various contexts of manipulation in virtual reality. We consider that such a TUI should be reconfigurable simply and quickly, to match many kinds of virtual objects, and to avoid interrupting the users' activity when being reconfigured. To enable single but also multiple-user manipulation, we believe that such a TUI should provide rigidity and act as haptic passive link between multiple hands.

For this purpose, we have designed a novel concept of TUI called the Reconfigurable Tangible Device (RTD) that is both rigid and reconfigurable. It proposes a generic and universal physical user interface made up of points rigidly linked together. The manipulation points are handles that form a simple shape that can be modified. 
The shape corresponding to the points roughly sketches the shape (or mesh) of the virtual object that is manipulated by the user(s). The RTD provides rigid, but easy-to-stretch, physical links between each manipulation point (or physical handles). The RTD has been designed to support two (or more) users in interacting together with the same virtual object. In this case, rigid links between users' hands act as a passive haptic feedback, which could improve the collaborative manipulation [Salzmann et al. 2009].

\subsection{RTD-3: Reconfigurable Tangible Device with 3 Points of Manipulation}

The first instance of RTD that we have developed is a triangular version called RTD-3 (Figure 1). This reconfigurable triangle is made up of three arms connected together by a pivot. As a result, it supports three points of manipulation (handles). It can be considered as a physical and reconfigurable version of the 3-Hand Manipulation technique introduced by Aguerreche et al. [Aguerreche et al. 2009]. This configuration fully determines the position and orientation of any attached virtual object through the positions of three non-aligned manipulation points placed on its surface.

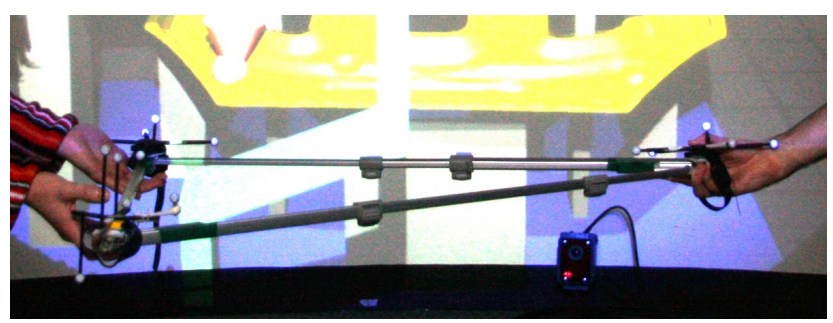

Figure 1: Reconfigurable Tangible Device with 3 points of manipulation (RTD-3): A reconfigurable triangle.

Each arm of the RTD-3 can be compressed or stretched (see Figure 2) by pulling a button to unlock/lock an arm. Varying the lengths of the arms and using many angles let users obtain small or large triangles with lengths from $38 \mathrm{~cm}$ up to $95 \mathrm{~cm}$, and angles varying from $20^{\circ}$ up to $130^{\circ}$. As a result, the RTD-3 can be used to match various basic shapes of virtual object (Figure 2). Users are able to grasp flat objects but also long, round or cubic objects. Virtual objects can be grasped horizontally or vertically.

\subsection{RTD-4: Reconfigurable Tangible Device with 4 Points of Manipulation}

The second instance of RTD that we have developed is using four points of manipulation (Figures 3 and 4). This instance is called RTD-4. This version enables users to compose various and complex shapes of $3 \mathrm{D}$ objects in $2 \mathrm{D}$ or $3 \mathrm{D}$, i.e., quadrilateral or tetrahedral (see Figure 3). It is made of four stretchable and rigid arms (same type as the RTD-3) connected together by articulated joints that enable the overall structure to become non-planar. This feature is extremely useful with articulated objects, such as a door to open for instance. An example of how to use the RTD-4 is given Figure 4 in which the RTD-4 approximately matches the shape of a virtual chair.

\subsection{Implementation details}

The two versions of the Reconfigurable Tangible Device introduced in this paper have been tested within a virtual reality center involving an ART $^{\circledR}$ optical tracking system. Virtual environment was displayed on a large stereoscopic screen. An optical marker was
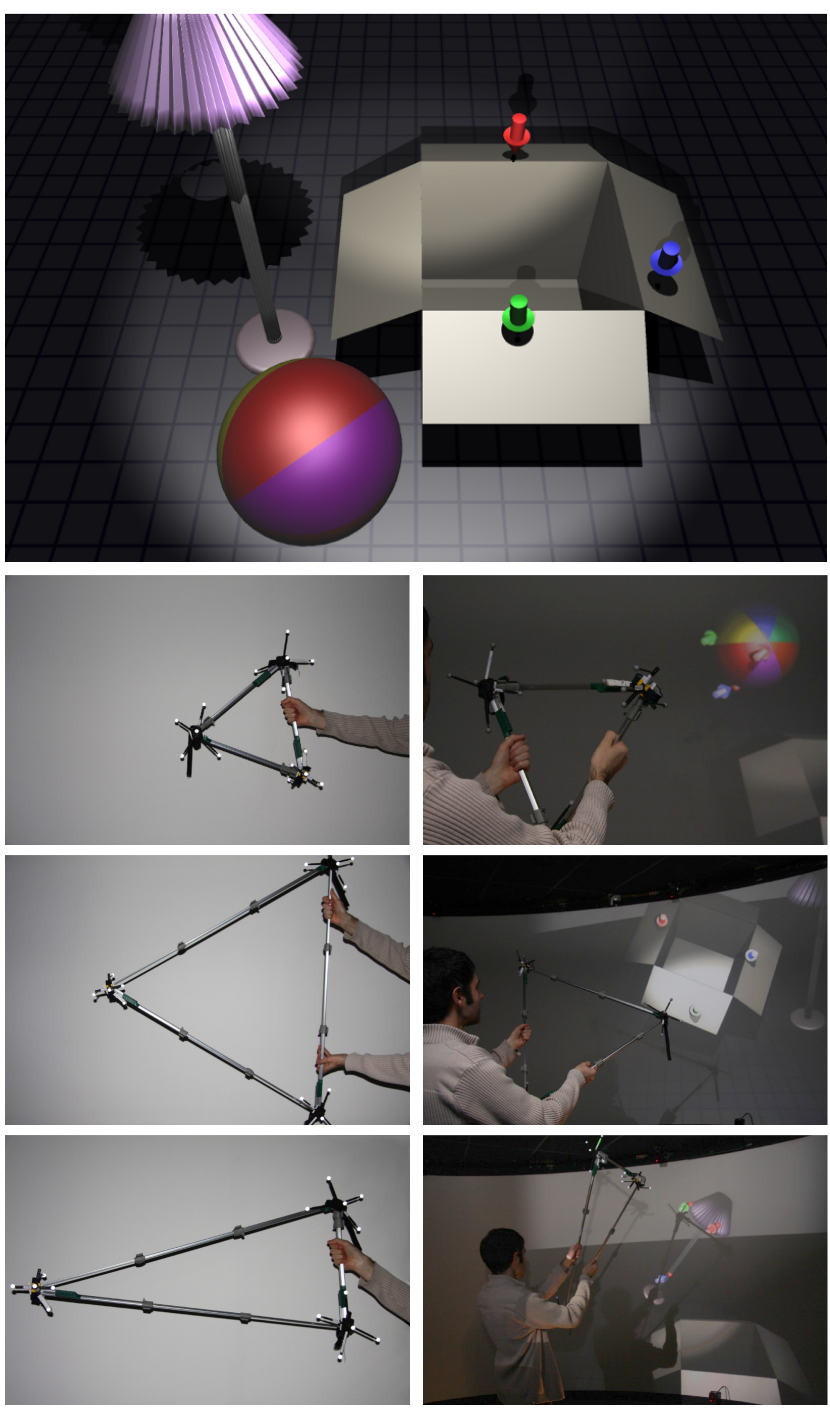

Figure 2: Different configurations of the Reconfigurable Tangible Device (RTD-3). The top picture provides an overview of the virtual scene. Other pictures show examples of configuration of the reconfigurable triangle (on the left) to match the manipulation of the corresponding objects (on the right).

placed on each handle (manipulation point) of the RTD. Infrared cameras were placed around users to track positions and orientations of the optical markers. Each marker was associated with a virtual pointer in the virtual environment.

We built both RTDs with low-cost camera tripods that we have disassembled. For RTD-3, the three telescopic arms of a tripod are linked together by hinges. For RTD-4, four tripods have been used: 4 arms and 4 tripod camera supports (or tripod 'heads'). Each tripod camera support provides 2 hinges which leads to RTD with many articulations that can be turned into 3D. The resulting RTDs are both rigid and light: $200 \mathrm{~g}$ for RTD-3 and $400 \mathrm{~g}$ for RTD-4.

In collaborative two-user situations, to manipulate the RTD-3, one user can put one hand on one corner while the other user puts their hands on the two remaining corners. They can move the triangle seamlessly together by applying movements to the device. The two users can naturally and easily use their two hands to hold the RTD-4 by all its corners. 

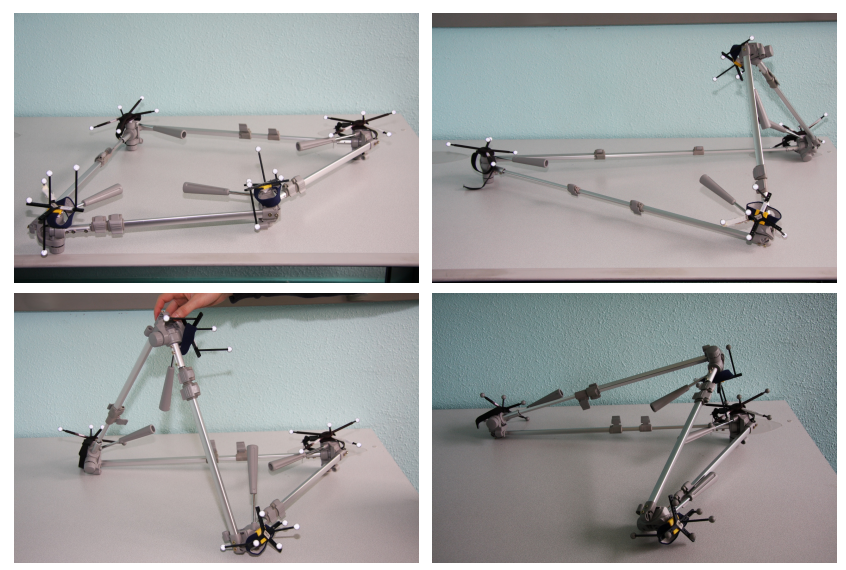

Figure 3: Reconfigurable Tangible Device with 4 points of manipulation (RTD-4): Different configurations and different shapes.
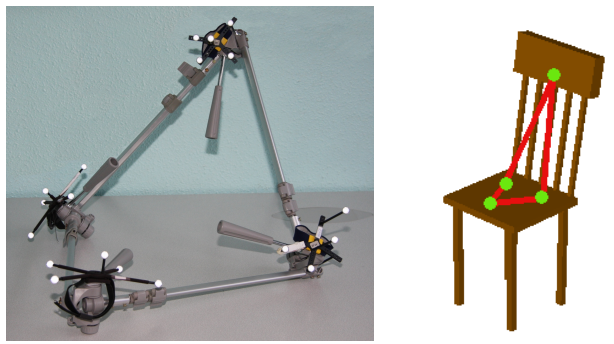

Figure 4: Example of configuration of the RTD-4 for manipulating a virtual chair.

When users want to attach the RTD to a virtual object they first set the relevant RTD shape, i.e. adjust the various lengths of the arms. They can then move the virtual pointers associated with the manipulation point of the device in order to touch the desired virtual object. After selecting the object (button click), users can begin to manipulate it.

\section{Manipulation examples}

In virtual reality, we can give different examples of manipulation in which our concept of Reconfigurable Tangible Device could be tested:

- Classical manipulation of 3D objects. The RTD is used to change the global position and orientation of the virtual object (classical case described above). Users can hook the RTD on any part of the object, provided that the RTD can be deformed to match the shape of this object. Movements of the RTD are directly transferred to the virtual object.

- Manipulation of articulated objects. The RTD is used to act on one part of the virtual object. Users associate the RTD with one part of the object that is linked with the other parts through an articulated joint. Movements of the RTD are transferred to the manipulated part of the object, and movements of other parts of the object are generated through the articulated joint.

- Deformation of objects. The RTD is used to modify and deform the shape of a virtual object (e.g., vertices of the 3D mesh). In this case, changing the shape of the RTD could modify the shape of the manipulated object using control points or direct manipulation of vertices of the mesh.

\section{Preliminary Evaluation}

As a preliminary evaluation of our concept, we mainly focused on the rigidity and ease of manipulation of RTD-3. We compared the use of RTD-3 with two classical techniques meant for collaborative virtual manipulation: (1) the Mean technique, and (2) the Separation of Degrees of Freedom (DoF). The task was a "pick-and-place" task involving two users in the manipulation and assembly of a virtual car hood model. Users were able to modify the shape of RTD-3 to appropriately match its shape with the shape of the virtual car hood model.

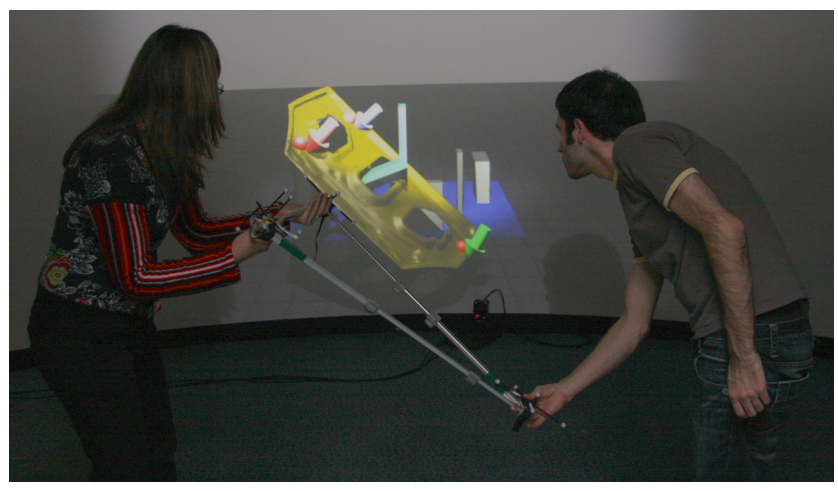

Figure 5: Experimental task: collaborative manipulation of a virtual car hood.

\subsection{Method}

24 participants volunteered to participate in our study (20 males, 4 females). They were computer-science students, software engineers or computer science researchers or lecturers. Users were positioned in front of a large screen with stereoscopic images $(3 \mathrm{~m} \times 2 \mathrm{~m})$. The tracking area of the ART infrared cameras was $4 \times 4 \mathrm{~m}$. Users wore shutter glasses and shared the same point of view (heads were not tracked).

First, users were shown the possibilities of the RTD-3 in terms of manipulation and reconfiguration. Then, users could adjust the shape of the RTD to fit the shape of their manipulated virtual object at their will, with no time constraint. Last, users had to move the virtual hood model outside a Z-shape. This Z-shape forces them to frequently rotate the hood to pass the Z-shape. Users had to coordinate their movements to translate and rotate the object (see Figure 5).

The RTD-3 was implemented as described above (see Figure 1). Then, the two other techniques were implemented as describe. The Mean technique [Ruddle et al. 2002] combines movements of the two users by averaging their changes in position and orientation. The Separation of DoF splits the control of the motion among users [Ruddle et al. 2002]: one user is manipulating translations only whilst the other one is responsible for rotations.

Experiments were conducted with 12 pairs of participants. Each pair of participant tested the 3 techniques. Participants were divided into 6 groups of users corresponding to the 6 orders of presentation of the 3 techniques. At the end of their testing, users rated the 3 techniques according to 5 criteria using a 7-point Likert scale: realism of the manipulation (Realism), feeling of presence in the virtual environment (Presence), training for the same task in the real world (Training), fatigue during the manipulation (Fatigue), and how much they liked a technique (Like). 


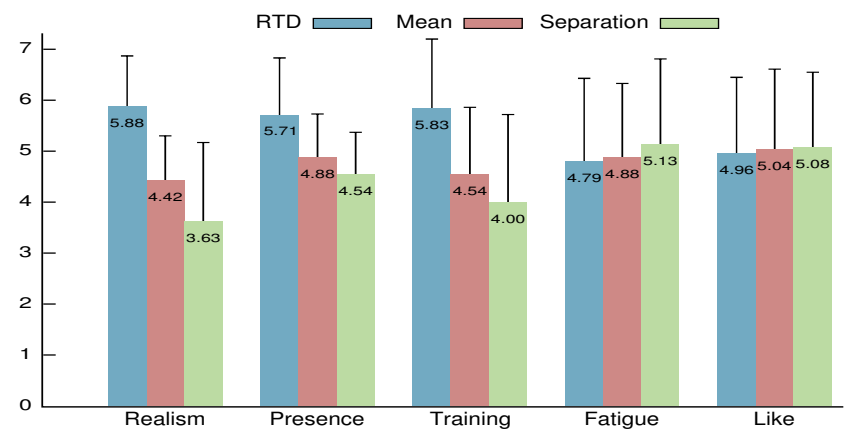

Figure 6: Mean and standard deviation for ratings of the different techniques using a 7-point Likert scale.

\subsection{Results}

Results for the ratings are given in Figure 6. An Analysis of Variance (global ANOVA) revealed that the technique used had a significant effect on the rating of the Realism rating $(F(2,69)=22.45$, $p<0.00001)$. For this criterion, the RTD was found significantly better rated than the Mean technique $(F(1,46)=29.02$, $p<0.00001)$, and than the Separation $(F(1,46)=35.71$, $p<0.00001)$. The ANOVA also revealed that the technique used had a significant effect on the Presence criterion $(F(2,69)=9.72$, $p=0.0002)$. For this criterion, the RTD was again found significantly better rated than the Mean technique $(F(1,46)=8.41, p=$ $0.0057)$, and Separation $(F(1,46)=16.73, p=0.0002)$. Last, the ANOVA also revealed that the technique used had a significant effect on the Training criterion $(F(2,69)=9.71, p=0.0002)$. For this criterion, the RTD was again found significantly better rated than the Mean technique $(F(1,46)=11.06, p=0.0017)$, and Separation $(F(1,46)=16.66, p=0.0002)$. No significant effects were found for the other criteria: Fatigue $(F(2,69)=0.03$, $p=0.85)$ and Like $(F(2,69)=0.04, p=0.9584)$.

Taken together the results of these preliminary tests suggest that our novel concept of a reconfigurable tangible device was preferred over classical non-tangible techniques used for collaborative manipulation of 3D objects in virtual environments. This first experiment must now be complemented by other and more quantitative experiments.

\section{Conclusion}

We have introduced the novel concept of a Reconfigurable Tangible Device for 3D object manipulation in virtual environments. The Reconfigurable Tangible Device is a physical interface that can be altered by the user to appropriately match the shape of a virtual object. It is made up of handles (or manipulation points) rigidly linked together by arms. This device can be reconfigured at any time as its arms can be compressed or stretched by users at will. The RTD can be used by multiple users, who can grasp different parts of the interface.

We have developed two versions of the RTD with three (RTD-3) or four (RTD-4) manipulation points. These two versions have been both implemented and tested within a virtual reality center for collaborative manipulation of 3D objects. They offer different manipulation possibilities with more or fewer manipulation points and more or fewer possibilities in terms of possible matching of $3 \mathrm{D}$ object shapes. A preliminary evaluation of our concept was conducted that compared the use of the RTD-3 with two classical techniques meant for collaborative manipulation in virtual environments: the
Mean technique, and the Separation of Degrees of Freedom (DoF). Our results suggest that the reconfigurable tangible devices were preferred by our participants providing them with a notably better sense of presence in the virtual environment.

Future Work concerns first more evaluations of our techniques, notably to compare the two versions of our reconfigurable tangible device (RTD-3 vs. RTD-4). We would also like to study the use of the RTD in different scenarios and use-cases such as object deformation by means of control points associated with the manipulation points of the RTD.

\section{References}

Aguerreche, L., Duval, T., AND LÉCuyer, A. 2009. 3-Hand Manipulation of Virtual Objects. In Proc. of JVRC 2009 (Joint Virtual Reality Conference of EGVE - ICAT - EuroVR), 153-156.

Anderson, D., Frankel, J. L., Marks, J., Leigh, D., SulLivan, E., Yedidia, J., AND RYALl, K. 1999. Building Virtual Structures with Physical Blocks. In Proc. of UIST, 71-72.

Bowman, D. A., KruijfF, E., LaViola, J. J., And Poupyrev, I. 2004. 3D User Interfaces: Theory and Practice. Addison Wesley.

Esposito, C., Paley, W. B., And Ong, J. 1995. Of Mice and Monkeys: A Specialized Input Device for Virtual Body Animation. In Proc. of I3D, 109-114.

FRAZER, J. H. 1995. An evolutionary architecture. Architectural Association, London.

Hinckley, K., Tullio, J., Pausch, R., Proffitt, D., And KASSELL, N. 1997. Usability analysis of 3d rotation techniques. In Proc. of UIST, 1-10.

ISHII, H., AND UlLmer, B. 1997. Tangible Bits: Towards Seamless Interfaces between People, Bits and Atoms. In Proc. of CHI, 234-241.

LeClerc, V., Parkes, A., And Ishit, H. 2007. Senspectra: A computationally augmented physical modeling toolkit for sensing and visualization of structural strain. In Proc. of CHI, 801804.

PARKes, A., LeClerc, V., AND IshiI, H. 2006. Glume: Exploring Materiality in a Soft Augmented Modular Modeling System. In Proc. of CHI, 1211-1216.

Ruddle, R. A., Savage, J. C. D., And Jones, D. M. 2002. Symmetric and Asymmetric Action Integration during Cooperative Object Manipulation in Virtual Environments. ACM Transactions on Computer-Human Interaction 9, 4, 285-308.

Salzmann, H., JaCOBS, J., AND Froehlich, B. 2009. Collaborative interaction in co-located two-user scenarios. In Proc. of JVRC 2009 (Joint Virtual Reality Conference of EGVE - ICAT EuroVR), 85-92.

Smith, R. T., Thomas, B. H., And PIekarski, W. 2008. Digital Foam Interaction Techniques for 3D Modeling. In Proc. of VRST, 61-68.

UlLmer, B., AND IshII, H. 2000. Emerging Frameworks for Tangible User Interfaces. IBM Systems Journal 39, 3-4, 915931.

Watanabe, R., Itoh, Y., Asai, M., Kitamura, Y., Kishino, F., AND KIKUCHI, H. 2004. The Soul of ActiveCube - Implementing a Flexible, Multimodal, Three-Dimensional Spatial Tangible Interface. Computers in Entertainment 2, 4, 15-15. 